\title{
Increased Activity of the Intracardiac Oxytocinergic System in the Development of Postinfarction Heart Failure
}

\author{
Agnieszka Wsol, ${ }^{1}$ Kaja Kasarello, ${ }^{1}$ Marek Kuch, ${ }^{2}$ \\ Kamila Gala, ${ }^{3}$ and Agnieszka Cudnoch-Jedrzejewska ${ }^{1}$ \\ ${ }^{1}$ Department of Experimental and Clinical Physiology, Laboratory of Centre for Preclinical Sciences, \\ Medical University of Warsaw, Warsaw, Poland \\ ${ }^{2}$ Department of Cardiology, Hypertension, and Internal Medicine, 2nd Medical Faculty, \\ Medical University of Warsaw, Warsaw, Poland \\ ${ }^{3}$ Department of Immunology, Transplantology and Internal Diseases, Transplantation Institute, \\ Medical University of Warsaw, Warsaw, Poland \\ Correspondence should be addressed to Agnieszka Wsol; awsol@wum.edu.pl
}

Received 15 July 2016; Revised 8 October 2016; Accepted 18 October 2016

Academic Editor: Ji-Fu Wei

Copyright (C) 2016 Agnieszka Wsol et al. This is an open access article distributed under the Creative Commons Attribution License, which permits unrestricted use, distribution, and reproduction in any medium, provided the original work is properly cited.

\begin{abstract}
Aim. The present study was designed to test the hypothesis that the development of postinfarction heart failure is associated with a change of activity of the intracardiac oxytocinergic system. Methods. Experiments were performed on male Sprague-Dawley rats subjected to myocardial infarction or sham surgery. Four weeks after the surgery, blood samples were collected and the samples of the left ventricle (LV) and right ventricle (RV) were harvested for evaluation of the mRNA expression (RT-PCR) of oxytocin (OT), oxytocin receptor (OTR), natriuretic peptides, and the level of OT and OTR protein (ELISA). The concentration of N-terminal $\mathrm{B}$-type natriuretic peptide was measured to determine the presence of heart failure. Results. Plasma NT-proBNP concentration was higher in the infarcted rats. In the infarcted rats, the expression of OT mRNA and the OT protein level were higher in the RV. There were no significant differences between infarcted and noninfarcted rats in the expression of OT mRNA and in the OT protein level in the fragments of the LV. In both the left and the right ventricles, OTR mRNA expression was lower but the level of OTR protein was higher in the infarcted rats. Conclusions. In the present study, we indicate that postinfarction heart failure is associated with an increased activity of the intracardiac oxytocinergic system.
\end{abstract}

\section{Introduction}

For a long time, oxytocin (OT), a neurohypophyseal hormone, was regarded as a pivotal factor in the stimulation of uterine contraction during labour, milk ejection, and the formation of maternal behaviour [1]. In recent years, we have observed how the spectrum of actions of oxytocin has conspicuously widened. Recent advances indicate that OT exerts an antidepressant and anxiolytic effect, and thus it may be a useful agent in the therapy of neuropsychiatric disorders such as autism, depression, social anxiety, and schizophrenia [2-5]. A growing body of evidence has shown that oxytocin plays a multiple role in the central and peripheral regulation of the cardiovascular function. The presence of oxytocin and its receptors was demonstrated in several brain regions involved in the regulation of blood pressure $[6,7]$. Several experimental and clinical studies established an OT function in the central and peripheral reduction of arterial blood pressure [8-11]. However, increases in blood pressure following OT central infusion have also been reported in normotensive Wistar-Kyoto rats, but the pressor effect in this case resulted from oxytocin administered in high doses binding to a Vla vasopressin receptor [12].

The mRNA for OT receptors was demonstrated not only in the central nervous system and in the uterus, but also in the atria, ventricles, caval veins, and the aorta. Further observation provided evidence for the heart and vasculature as sites of OT synthesis $[13,14]$. In the heart, OT was shown 
to increase the release of atrial natriuretic peptide (ANP) [15]. Recent studies emphasize the cardioprotective role of OT in experimental models of ischaemic cardiac injury [16-19]. In those studies, OT exerted a cardioprotective mechanism by stimulation of endothelial nitric oxide synthase-guanylate cyclase, ANP-cyclic guanosine monophosphate, phosphoinositide 3-kinase, and protein kinase $\mathrm{B}$ pathways and mild activation of mitochondrial ATP-dependent potassium channels $\left(\right.$ mito $\left._{\mathrm{ATP}}\right)$. Activation of the abovementioned processes by OT subsequently resulted in the reduction of the infarct size by the improvement of the following: the cardiac healing process and the remodelling ratio of cardiomyocytes and the interstitial matrix.

The rationale of the present study was to elucidate whether the development of postinfarction heart failure is associated with a change of activity of the oxytocinergic system in cardiomyocytes. Despite promising results of the role of OT in cardioprotection, the activity of the oxytocinergic system in the development of postmyocardial heart failure has not yet been investigated.

\section{Methods}

\subsection{Animals and Surgical Procedures}

2.1.1. Animals. All surgical and experimental protocols were conducted according to the international/EU guidelines and regulations on the use and care of laboratory animals. The experimental protocol was approved by the Second Local Animal Research Ethics Committee. All experiments were performed on 30 male Sprague-Dawley rats (SPRD/Möl/Lod, 250-350 g) bred in the Department of Animal Breeding. The rats were housed (2-3 animals per cage) under standard conditions including $12 \mathrm{~h} / 12 \mathrm{~h}$ light/dark cycle (light on at 7.00 a.m.) in a room with regulated temperature (range: $22-25^{\circ} \mathrm{C}$ ) and were fed a standard rat diet and allowed access to water ad libitum.

2.1.2. Myocardial Infarction/Sham Surgery. The animals were divided at random into two groups. One group was subjected to myocardial infarction $(n=20)$ and the other group to sham surgery $(n=10)$ at the age of $8-10$ weeks. Each of these procedures was performed under pentobarbital anaesthesia ( $5 \mathrm{mg} / 100 \mathrm{~g}$ body wt. i.p.; $20 \mu \mathrm{mol} / 100 \mathrm{~g}$ body wt.; Biowet Puławy). At the end of the surgical procedure, the animals were given an analgesic (buprenorphine chloride $3 \mu \mathrm{g} / 100 \mathrm{~g}$ body wt. i.p., $5.95 \mathrm{nmol} / \mathrm{mL}$, two times daily for 2-3 days) and an antibiotic (penicillin, Polfa $10000 \mathrm{IU} / 100 \mathrm{~g}$ body wt. i.m.; $0.047 \mathrm{mmol} / \mathrm{mL}$ ). After the surgical procedure, the rats were placed in separate home cages and remained under observation in the laboratory. After full recovery from anaesthesia, the animals were returned to the animal house.

The myocardial infarction was produced according to the technique described previously [20]. In brief, the rats were subjected to ligation of the left coronary artery just below the exit from the aorta after a surgical incision was made between the IV-V intercostal spaces. In the sham-operated rats, the cardiac pericardium was touched with a needle, but the coronary artery was not ligated. During the surgical procedure, the animals were ventilated by means of air puffs applied with a small rubber balloon. The rate of survival of the infarcted and the sham-operated rats was equal to $50 \%$ and $90 \%$, respectively. Of the animals that died, most died within the first 48 hours after surgery. For the molecular and biochemical measurements, tissue was derived from $n=10$ infarcted and $n=9$ sham-operated rats.

2.1.3. Tissue Harvesting and Postmortem Examination Procedures. Four weeks after the sham surgery or myocardial infarction, the rats were anaesthetised with pentobarbital sodium ( $5 \mathrm{mg} / 100 \mathrm{~g}$ body wt. i.p.; $20 \mu \mathrm{mol} / 100 \mathrm{~g}$ body wt.) and blood samples ( $2 \mathrm{~mL}$, EDTA) were collected. Then, each rat was sacrificed by decapitation. The heart was excised from the thorax, washed gently with saline, and placed on an iced surface. The ventricles were separated from the atria and the wall of the left ventricle (LV) including the septum was separated from the wall of the right ventricle (RV) along the longitudinal axis. Both ventricles were weighed. The tissue was placed flat on a piece of transparent millimetre paper. The external and internal circumferences of the LV (including the septum) and of the infarcted region were outlined. The surface of the infarct expressed as the number of square millimetres was determined on both sites and averaged. The dimension of the infarct scar was expressed as the percentage of the total left ventricle wall surface as described previously [20-22]. In the previous studies, this method of estimation provided the infarct size consistent with the infarct size obtained by histological examination [20, 22]. For further experiments, the fragments of the left ventricle muscle did not contain the myocardial infarction scar area. All tissue fragments were placed in liquid nitrogen immediately after excision and measuring. To avoid delay in the time of freezing tissues between the infarcted and sham-operated rats, all postmortem experimental processes were conducted by two operators simultaneously. One of the operators performed postmortem procedures on the sham-operated rats, while the other performed them on the infarcted rats. All tissues were frozen almost at the same time. Frozen tissue fragments and plasma were kept in deep freeze at $-85^{\circ} \mathrm{C}$.

2.2. Real-Time Polymerase Chain Reaction (PCR). Tissue samples were homogenized at a frequency of $25 \mathrm{~Hz}$ for 5 minutes in a TissueLyser (Qiagen GmbH, Hilden, Germany) homogenizer. Isolation of total RNA was performed automatically on a BioRobot EZ1 using the EZ1 RNA Universal Tissue Kit (Qiagen, Germany) according to the manufacturer's instructions (Qiagen GmbH, Hilden, Germany). RNA concentration and purity were assessed with a NanoDrop spectrophotometer at $260 \mathrm{~nm}$ (ND-1000 Spectrophotometer, Thermo Fisher Scientific Inc.).

Real-time PCR was performed on a ViiA ${ }^{\mathrm{TM}} 7$ Real-Time PCR System using TaqMan ${ }^{\circledR}$ RNA-to- $\mathrm{C}_{\mathrm{T}}{ }^{\text {TM }}$ 1-Step Kit (Applied Biosystems, Foster City, USA). Specific primer and probe sets were purchased from Applied Biosystems: oxytocin (Rn00564446_g1), oxytocin receptor (Rn00563503_m1), ANP (Rn00664637_g1), and BNP (Rn00580641_ml) (Table 1). 
TABLE 1: Sequences of oligonucleotides used in the present study.

\begin{tabular}{lccc}
\hline Gene & Sense primer $\left(5^{\prime}-3^{\prime}\right)$ & Antisense primer $\left(5^{\prime}-3^{\prime}\right)$ & Accession number \\
\hline OT, Oxt & GACGGTGGATCTCGGACTGAA & CGCCCCTAAAGGTATCATCACAAA & Rn00564446_g1 \\
OTR, Oxtr & GTCAATGCGCCCAAGGAAG & GATGCAAACCAATAGACACC & Rn00563503_m1 \\
ANP, $N p p a$ & CAGCATGGGCTCCTTCTCCA & GTCAATCCTACCCCCGAAGCAGCT & Rn00664637_g1 \\
BNP, $N p p b$ & CCATCGCAGCTGCCTGGCCCATCACT & GACTGCGCCGATCCGGTC & Rn00580641_m1 \\
\hline
\end{tabular}

BNP: B-type natriuretic peptide; OT: oxytocin; OTR: oxytocin receptor.

Glyceraldehyde-3-phosphate dehydrogenase (GAPDH; $4352338 \mathrm{E}$ ) was used as a housekeeping gene. Total reaction volume was $50 \mu \mathrm{L}$. The experiment was conducted using MicroAmp ${ }^{\mathrm{TM}}$ Optical 96-Well Reaction Plates with Barcode (Applied Biosystems). Amplification was performed in 40 cycles at $95^{\circ} \mathrm{C}$ for 15 seconds and at $60^{\circ} \mathrm{C}$ for 1 minute. Duplicates of each sample were performed. The $\mathrm{Ct}$ (threshold cycle) for the target gene and the Ct for the internal control were determined for each sample. The relative gene expression was given on the basis of estimations of the values of the delta cycle threshold $(\Delta \mathrm{Ct})$ by relative quantification to the endogenous control.

\subsection{Enzyme-Linked Immunosorbent Assay (ELISA). The fol-} lowing EIA Kits were used for the evaluation of oxytocin, oxytocin receptor concentration in the heart's homogenates, and N-terminal prohormone B-type natriuretic peptide (NTproBNP) in plasma: Oxytocin EIA Kit (Phoenix Pharmaceuticals Inc., USA), Oxytocin Receptor EIA Kit (Sunred Biological Technology Co. Ltd., China), and NT-proBNP (Wuhan EIAab Science Co. Ltd., China). For the ventricle cardiac muscle, each sample of tissue was homogenized in the TissueLyser bead mixer (Qiagen, USA) and centrifuged $\left(10000 \mathrm{rpm}\right.$ for 10 minutes, $\left.4^{\circ} \mathrm{C}\right)$. The supernatant was collected and frozen at $-80^{\circ} \mathrm{C}$ until analysis. Total protein concentration was measured using bicinchoninic acid (BCA) Protein Assay Kit (Pierce, Holland), according to the manufacturer's instructions. Results obtained were presented as an absolute ratio: concentration/total protein concentration $\left(\times 10^{-9}\right)$ for OT and OTR and total protein concentration $(\mathrm{pg} / \mathrm{mL})$ for plasma NT-proBNP.

2.4. Statistical Analysis. All values presented in the text and figures are means \pm SE. STATISTICA software (version 10) was used for statistical analysis of the data. One-way ANOVA followed by the Tukey post hoc test was applied for the parameters with normal distribution (OT, OTR, BNP, and ANP mRNA expression, left and right ventricular mass, and body mass). Parameters with nonnormal distribution (OT, OTR protein level, and NT-proBNP plasma concentration) were analyzed with the Kruskal-Wallis test with Dunn's multiple comparison nonparametric test. The differences were considered significant if $P<0.05$.

\section{Results and Discussion}

3.1. Results. Total body mass in the infarcted and shamoperated rats was equal to $287.5 \pm 12.1 \mathrm{~g}$ and $290.5 \pm 6.1 \mathrm{~g}$, respectively, and did not differ significantly. We did not find significant differences between the masses of the left ventricle in infarcted and sham-operated rats $(0.22 \pm 0.004$ versus $0.24 \pm 0.01 \mathrm{~g})$. Right ventricle mass was significantly higher in the infarcted rats $(0.08 \pm 0.004$ versus $0.06 \pm 0.002 \mathrm{~g}$ $[F(1,17)=6.417 ; P<0.05])$. The infarct size was $47.3 \pm 5.74 \%$ of the left ventricle surface.

Plasma NT-proBNP concentration was significantly higher in the infarcted rats (Figure 1(a)). In the left ventricle, infarcted rats manifested significantly higher expression for BNP mRNA $[F(1,15)=428.15 ; P<0.001]$ and ANP mRNA $[F(1,13)=323.95 ; P<0.001]$ (Figures $1(\mathrm{~b})$ and $1(\mathrm{c})$ ).

In the infarcted rats, expression of OT mRNA $[F(1,14)=$ 5.699; $P<0.05$ ] and OT protein level were higher in the right ventricle $(P<0.05)$. There were no significant differences between infarcted and noninfarcted rats in the expression of OT mRNA and in OT protein in the fragments of the left ventricle (Figures 2(a) and 2(b)).

We found that the OTR mRNA expression was significantly lower in the infarcted rats both in the left ventricle $[F(1,12)=353.75 ; P<0.001]$ and in the right ventricle $[F(1,12)=73.833 ; P<0.001]$, whereas the level of OTR protein was higher in the infarcted rats in both the left and the right ventricles (Figures 3(a) and 3(b)).

3.2. Discussion. In the present study, we show for the first time that postinfarction heart failure is associated with significant changes in both the mRNA expression and the protein level of the intracardiac oxytocin and oxytocin receptor. By analyzing the results of protein expression for both OT and OTR, we conclude that the experimental model of postinfarct heart failure was associated with an increase in activity of the heart's oxytocinergic system.

Results of past studies revealed that OT increases the release of natriuretic peptide, a powerful marker of the left ventricle systolic function, from the heart [23-25]. Repeated injections of OT in rats resulted in both an increase of natriuresis and elevation of plasma ANP. Moreover, the stimulation of ANP release by OT was observed also in the absence of neuronal influence in experiments with isolated heart perfusion, which provided evidence for ANP release by the intracardiac oxytocinergic system [15]. In the present study, we show by means of an increase in the expression of ANP, BNP mRNA, and plasma NT-proBNP concentration that our experimental model resulted in the development of heart failure. At the same time, we observed an increase in OT level in the muscle of the right ventricle and an increase in protein synthesis for OTR in both ventricles 


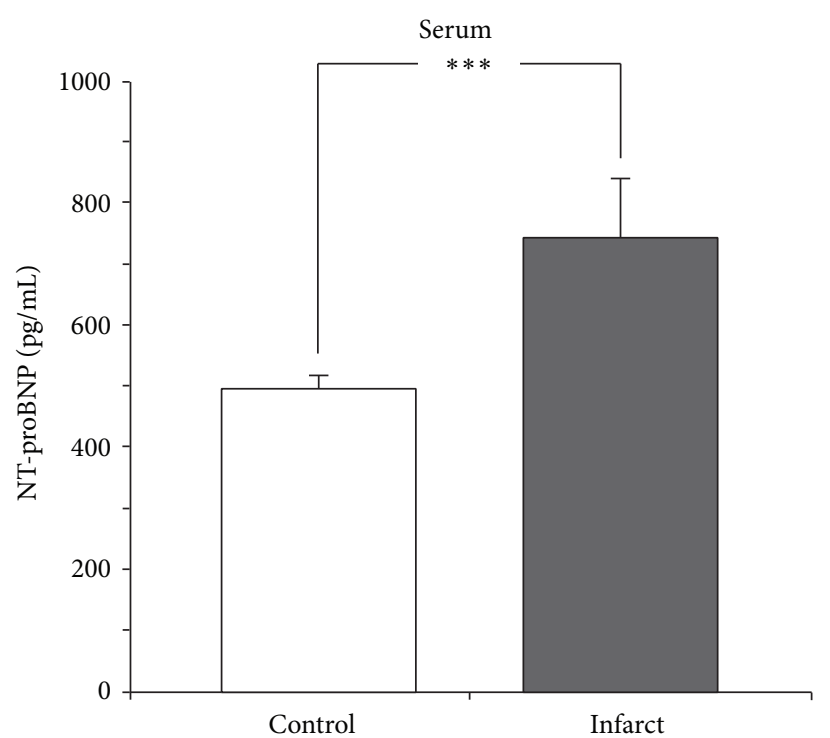

(a)

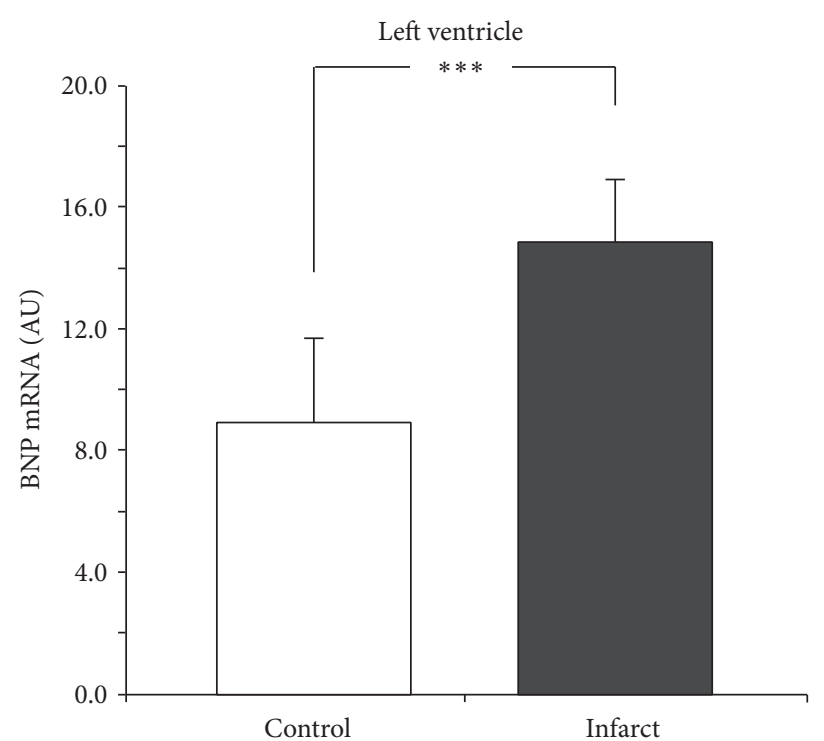

(b)

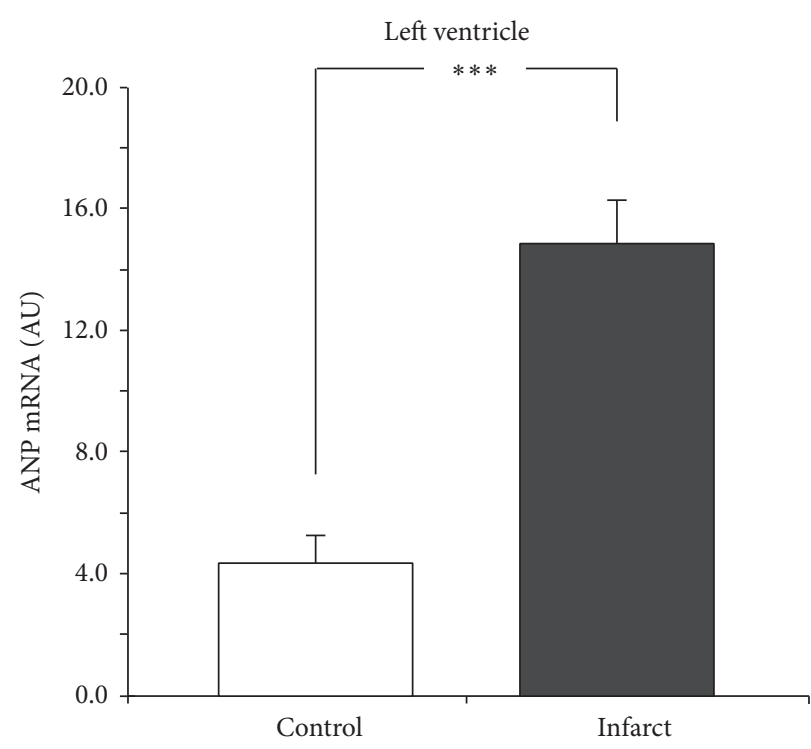

(c)

FIGURE 1: Serum NT-proBNP concentration (a) and average expression of BNP mRNA (b) and ANP mRNA (c) in the fragments of the left ventricle muscle in the sham-operated rats (control) and in rats with myocardial infarction (infarct). Arbitrary units: relative gene expression was given on the basis of estimations of the values of the delta cycle threshold $(\Delta \mathrm{Ct})$ by relative quantification to the endogenous control. Means \pm standard errors are shown. Significant differences between the experimental groups: ${ }^{* * *} P<0.001$.

of the heart. Jankowski et al., in the experimental model of myocardial infarction in rats, reported the reduction of natriuretic peptides by OT infusions as a result of left ventricle systolic function improvement [17]. These results are questionable as, in most of the studies, OT was shown to increase natriuretic peptides (ANP) production $[15,24,25]$. According to the data presented from experimental studies and the results from our observations, we may suggest that an increase in natriuretic peptide in heart failure could result from the increased activity of intracardiac OT. However, further investigations to assess the direct correlation between intracardiac OT activation and natriuretic peptides expression in our experimental model of postinfarct heart failure are needed.

It should be emphasized that in our study we showed, for the first time, changes in the expression of OT and OTR mRNA and the level of OT and OTR protein four weeks after myocardial infarction, which was reported previously as sufficient time for the development of postinfarct heart failure [21, 22]. Most of the up-to-date studies on the role of oxytocin in myocardial infarction were based on experimental methods involving an ischaemia-reperfusion model in vitro $[19,26,27]$ or in vivo $[16,17]$. In the study by Jankowski et al., myocardial infarction was evoked by chronic ligation of the left anterior 


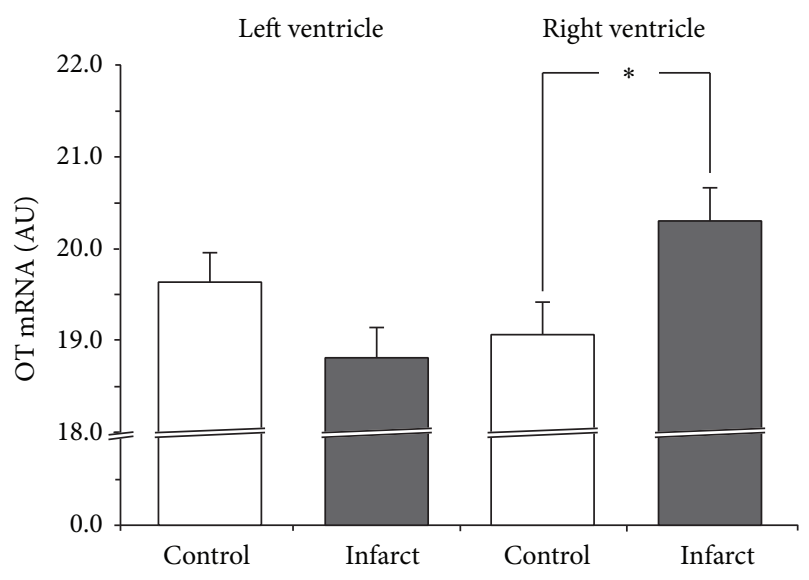

(a)

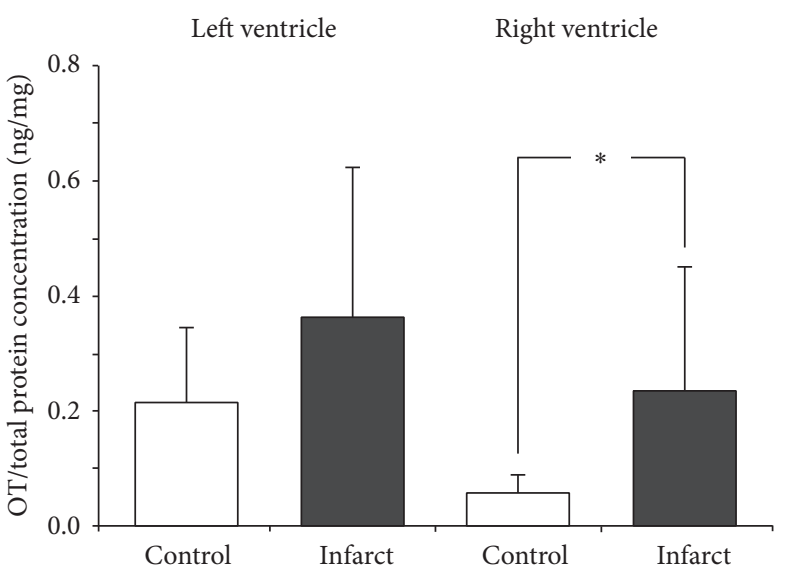

(b)

Figure 2: Average expression of oxytocin (OT) mRNA (a) and protein (b) in the left and right ventricle muscles in the sham-operated rats (control) and in rats with myocardial infarction (infarct). Arbitrary units: relative gene expression was given on the basis of estimations of the values of the delta cycle threshold $(\Delta \mathrm{Ct})$ by relative quantification to the endogenous control. Oxytocin concentration presented as an absolute ratio: oxytocin/total protein concentration. Means \pm standard errors are shown. Significant differences between the experimental groups: ${ }^{*} P<0.05$.

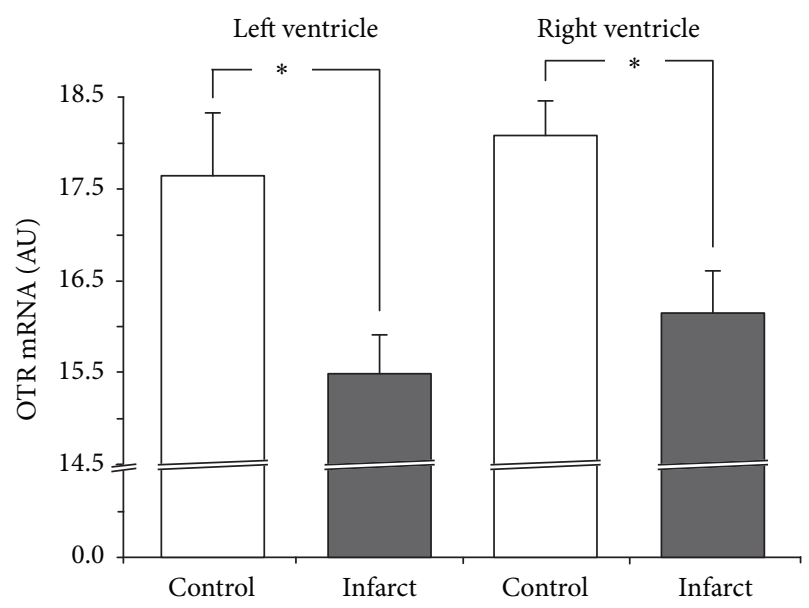

(a)

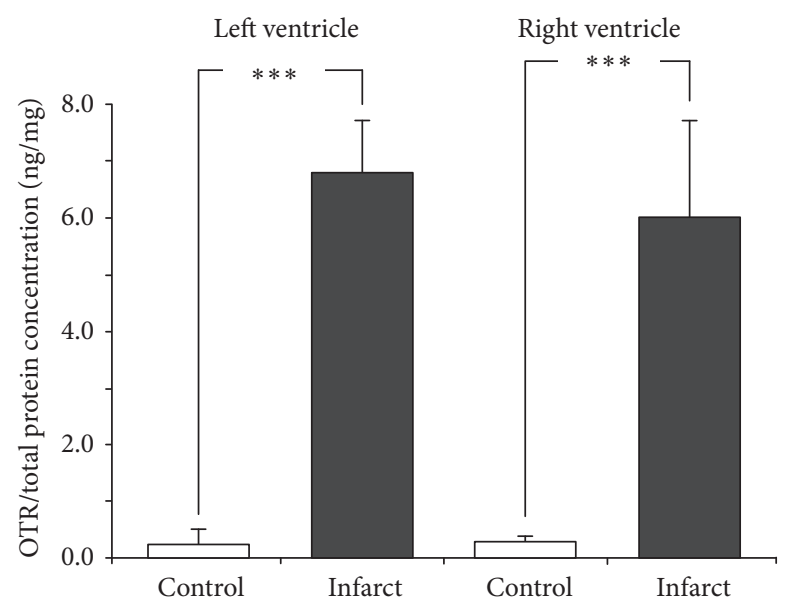

(b)

FIGURE 3: Average expression of oxytocin receptor (OTR) mRNA (a) and protein (b) in the left and right ventricle muscles in the shamoperated rats (control) and in rats with myocardial infarction (infarct). Arbitrary units: relative gene expression was given on the basis of estimations of the values of the delta cycle threshold $(\Delta \mathrm{Ct})$ by relative quantification to the endogenous control. Oxytocin receptor concentration presented as an absolute ratio: oxytocin/total protein concentration. Means \pm standard errors are shown. Significant differences between the experimental groups: ${ }^{*} P<0.05 ;{ }^{* * *} P<0.001$.

descending coronary artery [17]. In that study, the time of observation was limited only to 7 days after myocardial infarction. Lower OTR mRNA expression observed here is consistent with the results published previously by Jankowski et al. [17]. Nevertheless, the authors of the study reported a reduction in OTR protein in the fragments of the left ventricle, 3 and 7 days after myocardial infarction. The cardiac muscle was not examined in that study for OT content. The increase in OTR protein reported here is observed at a later phase of infarct. This raises an important question of whether the differences between OTR protein level obtained in our study and those in the abovementioned study by Jankowski et al. could result from the changes in OT protein level in tissue samples in the early period after myocardial infarction in comparison with chronic postinfarct heart failure.

In our study, we observed a decrease of OTR mRNA while the OTR protein level in the ventricles of the infarcted rats increased four weeks after myocardial infarction. A suggested mechanism(s) of the observed dissociation between OTR mRNA and its protein may involve desensitization and internalization of OTRs or changes in the regulation of synthesis and degradation of mRNA. Additionally, the dissociation may be disease-dependent, particularly during the progression of heart failure. It has been shown that, in HEK293 cells, 
internalization of OTR occurs mainly due to the clathrindependent pathway, but another mechanism involved in the internalization of OTR into cellular membrane caveolae has been proposed [28]. It is possible that the increased content of OTR observed in our study also involves the population of caveolized OTR inaccessible for oxytocin. Previous studies showed that exposure to oxytocin in human myometrial cells resulted in a 10-fold reduction of oxytocin binding capacity. Moreover, in that experiment, total OTR amount did not change in the first 48 hours after exposure to OT, and OTRs were not internalized in this process. At the same time, OTR mRNA was reduced [29].

In the present study, we found higher expression of OT mRNA and higher protein levels in the fragments of the right but not in the left ventricle. The data from previous studies indicate that the OT concentration is the highest in the right atrium in comparison with the left atrium and in the right ventricle in comparison with the left ventricle cardiomyocytes [13]. This observation suggests that the structures in the "right heart" are more actively synthesizing oxytocin. On the other hand, in our results, we found an increase only in the right ventricle mass, which also signalizes right ventricle hypertrophy, 4 weeks after the left coronary artery ligation. A plethora of evidence showed that stimulation with estrogens increases oxytocin synthesis [30-32]. Activation of estrogen receptors $\beta$ was shown to raise the amount of oxytocin peptide [29]. Moreover, in the study by Cavasin et al., estrogens prevented deterioration of the cardiac function and remodelling in the experimental model of myocardial infarction [33]. Therefore, there is another suggested mechanism that could influence the lack of oxytocin mRNA: protein expression in the left ventricle observed in our study may be correlated with a possible change in estrogen activity (especially via estrogen receptor $\beta$ ) in the development of left ventricle remodelling due to myocardial infarction.

The results from the present study may suggest an increased activity of the oxytocinergic system in the heart as another aspect of the OT cardioprotective function in the experimental model of myocardial infarction. Results from recent studies indicate that increased activity of the oxytocinergic system in experimental models of left ventricle damage may slow down the progression of heart failure due to the reduction of the infarct size and cardiac remodelling. In the study by Jankowski et al., the treatment with OT chronic infusions over 7 days following myocardial infarction resulted in the improvement of the left ventricle systolic function, reduction of apoptosis, expression of proinflammatory cytokines transcripts, and inflammatory cell migration in the infarct region [17]. The authors of that study did not measure oxytocin mRNA expression and protein level at the same time; thus, it cannot be excluded that endogenous intracardiac OT could be partially responsible for the observed cardioprotective effects. In the other study, pretreatment with OT before ischaemia-reperfusion exposure resulted in the reduction of infarct size and activation of the prosurvival p38-MAPK and Akt-kinase pathways [26, 27]. In the in vitro model of ischaemia-reperfusion-induced myocardial damage, Ondrejcakova et al. showed that exposure to OT prior to ischaemia resulted in the reduction of the infarct size due to the negative chronotropic effect [19].

\section{Conclusions}

In conclusion, the main finding of the present study is that postmyocardial infarction heart failure is associated with an increased activity of the intracardiac oxytocinergic system. As oxytocin was found as a novel and important cardioprotective factor in myocardial ischaemia and was also shown to increase natriuretic peptide release, we therefore suggest that increased activity of the intracardiac oxytocinergic system after left ventricle damage may have an inhibitory impact on the progression of heart failure. According to the importance of this novel issue in the pathophysiology of heart failure, further investigations on oxytocin in heart failure are incontestably necessary.

\section{Competing Interests}

The authors report no financial or other competing interests relevant to the subject of this publication.

\section{Acknowledgments}

The authors would like to express their gratitude to Mrs. Małgorzata Kowalczyk for her technical assistance and to Mr. Marcin Kumosa for the preparation of the illustrations. This study was supported by grants from the Warsaw Medical University (1MA/PM12/14/14; 1MAW2/2015).

\section{References}

[1] R. Ivell and J. A. Russell, "Oxytocin: cellular and molecular approaches in medicine and research," Reviews of Reproduction, vol. 1, pp. 13-18, 1996.

[2] A.-M. Bao, G. Meynen, and D. F. Swaab, “The stress system in depression and neurodegeneration: focus on the human hypothalamus," Brain Research Reviews, vol. 57, no. 2, pp. 531553, 2008.

[3] I. D. Neumann and R. Landgraf, "Balance of brain oxytocin and vasopressin: implications for anxiety, depression, and social behaviors," Trends in Neurosciences, vol. 35, no. 11, pp. 649-659, 2012.

[4] D. M. Cochran, D. Fallon, M. Hill, and J. A. Frazier, "The role of oxytocin in psychiatric disorders: a review of biological and therapeutic research findings," Harvard Review of Psychiatry, vol. 21, no. 5, pp. 219-247, 2013.

[5] E. Anagnostou, L. Soorya, J. Brian et al., "Intranasal oxytocin in the treatment of autism spectrum disorders: a review of literature and early safety and efficacy data in youth," Brain Research, vol. 1580, pp. 188-198, 2014.

[6] C. Barberis and E. Tribollet, "Vasopressin and oxytocin receptors in the central nervous system," Critical Reviews in Neurobiology, vol. 10, no. 1, pp. 119-154, 1996.

[7] M. L. Boccia, P. Petrusz, K. Suzuki, L. Marson, and C. A. Pedersen, "Immunohistochemical localization of oxytocin receptors in human brain," Neuroscience, vol. 253, pp. 155-164, 2013. 
[8] J. B. Sartain, J. J. Barry, P. W. Howat, D. I. McCormack, and M. Bryant, "Intravenous oxytocin bolus of 2 units is superior to 5 units during elective Caesarean section," British Journal of Anaesthesia, vol. 101, no. 6, pp. 822-826, 2008.

[9] A. Wsł, A. Cudnoch-Jedrzejewska, E. Szczepanska-Sadowska, S. Kowalewski, and J. Dobruch, "Central oxytocin modulation of acute stress-induced cardiovascular responses after myocardial infarction in the rat," Stress, vol. 12, no. 6, pp. 517-525, 2009.

[10] J. Gutkowska, Y. Aliou, J. L. Lavoie, K. Gaab, M. Jankowski, and T. L. Broderick, "Oxytocin decreases diurnal and nocturnal arterial blood pressure in the conscious unrestrained spontaneously hypertensive rat," Pathophysiology, vol. 23, no. 2, pp. 111121, 2016.

[11] M. Petersson and K. Uvnäs-Moberg, "Effects of an acute stressor on blood pressure and heart rate in rats pretreated with intracerebroventricular oxytocin injections," Psychoneuroendocrinology, vol. 32, no. 8-10, pp. 959-965, 2007.

[12] A. Wsol, E. Szczepanska-Sadowska, S. Kowalewski, L. Puchalska, and A. Cudnoch-Jedrzejewska, "Oxytocin differently regulates pressor responses to stress in WKY and SHR rats: the role of central oxytocin and V1a receptors," Stress, vol. 17, no. 1, pp. 117-125, 2014.

[13] M. Jankowski, F. Hajjar, S. Al Kawas et al., "Rat heart: a site of oxytocin production and action," Proceedings of the National Academy of Sciences of the United States of America, vol. 95, no. 24, pp. 14558-14563, 1998.

[14] M. Jankowski, D. Wang, F. Hajjar, S. Mukaddam-Daher, S. M. McCann, and J. Gutkowska, "Oxytocin and its receptors are synthesized in the rat vasculature," Proceedings of the National Academy of Sciences of the United States of America, vol. 97, no. 11, pp. 6207-6211, 2000.

[15] J. Gutkowska, M. Jankowski, C. Lambert, S. Mukaddam-Daher, H. H. Zingg, and S. M. McCann, "Oxytocin releases atrial natriuretic peptide by combining with oxytocin receptors in the heart," Proceedings of the National Academy of Sciences of the United States of America, vol. 94, no. 21, pp. 11704-11709, 1997.

[16] A. M. Alizadeh, M. Faghihi, H. R. Sadeghipour, F. MohammadGhasemi, and V. Khori, "Role of endogenous oxytocin in cardiac ischemic preconditioning," Regulatory Peptides, vol. 167, no. 1, pp. 86-90, 2011.

[17] M. Jankowski, V. Bissonauth, L. Gao et al., "Anti-inflammatory effect of oxytocin in rat myocardial infarction," Basic Research in Cardiology, vol. 105, no. 2, pp. 205-218, 2010.

[18] M. Faghihi, A. M. Alizadeh, V. Khori, M. Latifpour, and S. Khodayari, "The role of nitric oxide, reactive oxygen species, and protein kinase $\mathrm{C}$ in oxytocin-induced cardioprotection in ischemic rat heart," Peptides, vol. 37, no. 2, pp. 314-319, 2012.

[19] M. Ondrejcakova, T. Ravingerova, J. Bakos, D. Pancza, and D. Jezova, "Oxytocin exerts protective effects on in vitro myocardial injury induced by ischemia and reperfusion," Canadian Journal of Physiology and Pharmacology, vol. 87, no. 2, pp. 137$142,2009$.

[20] J. Dobruch, A. Cudnoch-Jedrzejewska, and E. SzczepanskaSadowska, "Enhanced involvement of brain vasopressin V1 receptors in cardiovascular responses to stress in rats with myocardial infarction," Stress, vol. 8, no. 4, pp. 273-284, 2005.

[21] F. H. H. Leenen, B. Yuan, and B. S. Huang, "Brain 'ouabain' and angiotensin II contribute to cardiac dysfunction after myocardial infarction," American Journal of Physiology-Heart and Circulatory Physiology, vol. 277, no. 5, pp. H1786-H1792, 1999.
[22] A. Cudnoch-Jedrzejewska, E. Szczepanska-Sadowska, J. Dobruch et al., "Differential sensitisation to central cardiovascular effects of angiotensin II in rats with a myocardial infarct: relevance to stress and interaction with vasopressin," Stress, vol. 11, no. 4, pp. 290-301, 2008.

[23] R. Ghashghaei, B. Arbit, and A. S. Maisel, "Current and novel biomarkers in heart failure: bench to bedside," Current Opinion in Cardiology, vol. 31, no. 2, pp. 191-195, 2016.

[24] M. A. Haanwinckel, L. K. Elias, A. L. V. Favaretto, J. Gutkowska, S. M. Mccann, and J. Antunes-Rodrigues, "Oxytocin mediates atrial natriuretic peptide release and natriuresis after volume expansion in the rat," Proceedings of the National Academy of Sciences of the United States of America, vol. 92, no. 17, pp. 79027906, 1995.

[25] T. J. Soares, T. M. Coimbra, A. R. Martins et al., "Atrial natriuretic peptide and oxytocin induce natriuresis by release of cGMP," Proceedings of the National Academy of Sciences of the United States of America, vol. 96, no. 1, pp. 278-283, 1999.

[26] M. Ondrejcakova, M. Barancik, M. Bartekova, T. Ravingerova, and D. Jezova, "Prolonged oxytocin treatment in rats affects intracellular signaling and induces myocardial protection against infarction," General Physiology and Biophysics, vol. 31, no. 3, pp. 261-270, 2012.

[27] A. Gonzalez-Reyes, A. Menaouar, D. Yip et al., "Molecular mechanisms underlying oxytocin-induced cardiomyocyte protection from simulated ischemia-reperfusion," Molecular and Cellular Endocrinology, vol. 412, pp. 170-181, 2015.

[28] G. Gimpl, K. Burger, E. Politowska, J. Ciarkowski, and F. Fahrenholz, "Oxytocin receptors and cholesterol: interaction and regulation," Experimental Physiology, vol. 85, pp. 41S-49S, 2000.

[29] G. Gimpl and F. Fahrenholz, "The oxytocin receptor system: structure, function, and regulation," Physiological Reviews, vol. 81, no. 2, pp. 629-683, 2001.

[30] S. Phaneuf, G. Asbóth, M. P. Carrasco et al., "The desensitization of oxytocin receptors in human myometrial cells is accompanied by down-regulation of oxytocin receptor messenger RNA," Journal of Endocrinology, vol. 154, no. 1, pp. 7-18, 1997.

[31] B. F. Mitchell and R. Chibbar, "Synthesis and metabolism of oxytocin in late gestation in human decidua," Advances in Experimental Medicine and Biology, vol. 395, pp. 365-380, 1995.

[32] L. J. Young, S. Muns, Z. Wang, and T. R. Insel, "Changes in oxytocin receptor mRNA in rat brain during pregnancy and the effects of estrogen and interleukin-6," Journal of Neuroendocrinology, vol. 9, no. 11, pp. 859-865, 1997.

[33] M. A. Cavasin, S. S. Sankey, A.-L. Yu, S. Menon, and X.P. Yang, "Estrogen and testosterone have opposing effects on chronic cardiac remodeling and function in mice with myocardial infarction," American Journal of Physiology-Heart and Circulatory Physiology, vol. 284, no. 5, pp. H1560-H1569, 2003. 


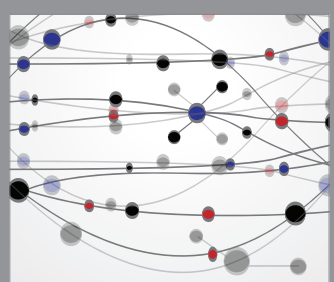

The Scientific World Journal
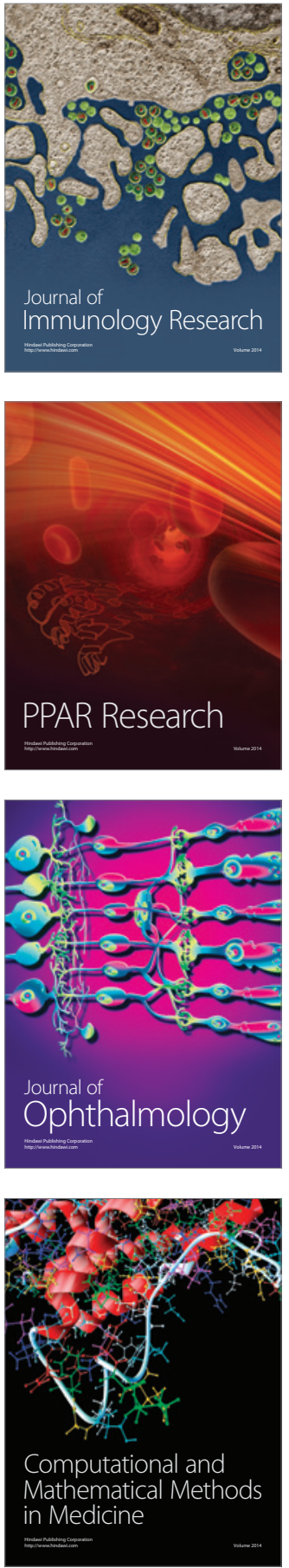

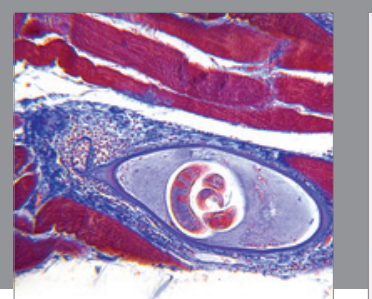

Gastroenterology Research and Practice

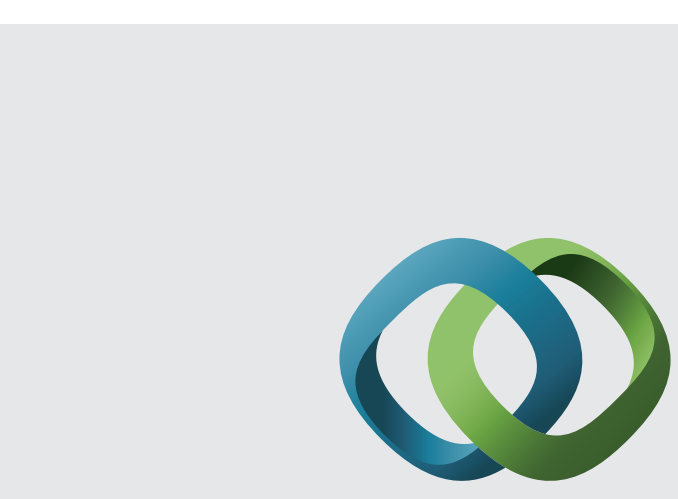

\section{Hindawi}

Submit your manuscripts at

http://www.hindawi.com
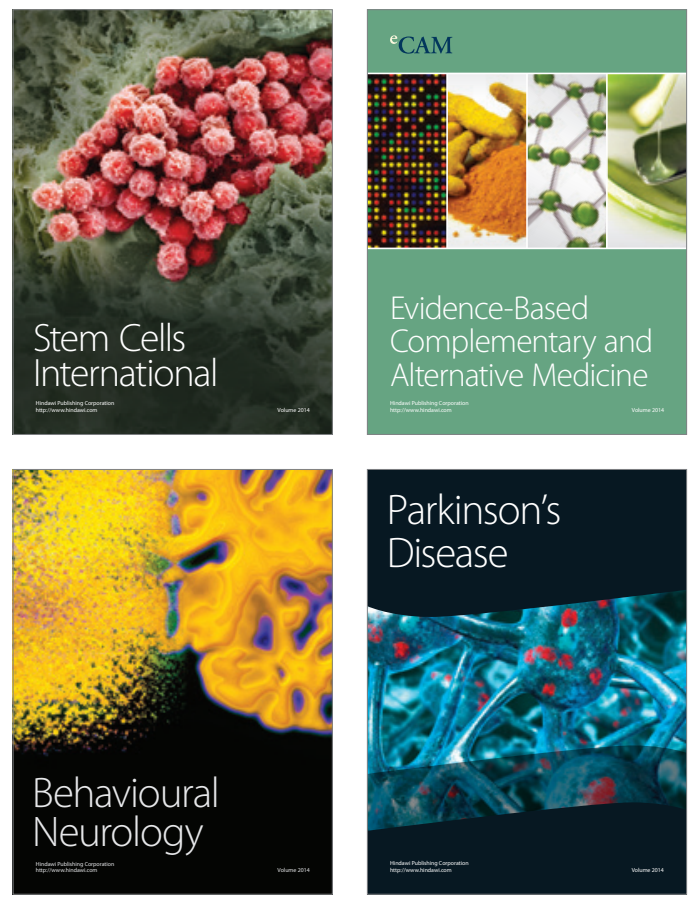
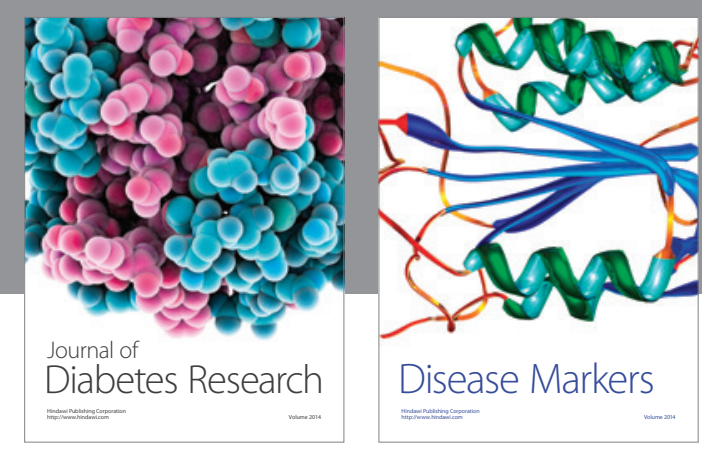

Disease Markers
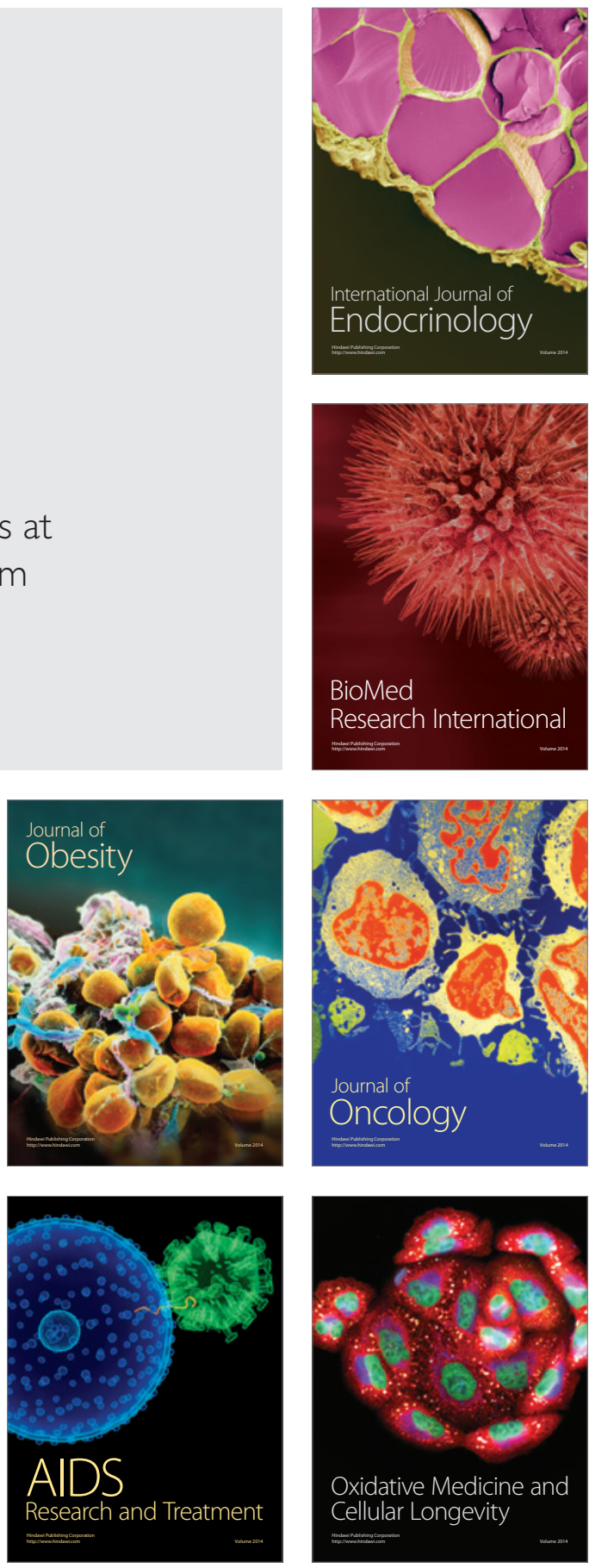